\title{
HE'S VARIATIONAL ITERATION METHOD FOR SOLVING MULTI-DIMENSIONAL OF NAVIER STOKES EQUATION
}

\section{MOHAMED ZELLAL ${ }^{1,2, *}$, KACEM BELGHABA ${ }^{2}$}

\author{
${ }^{1}$ Department of Common Core in Exact Sciences and Informatics, Faculty of Exact Sciences and \\ Informatics, Hassiba Benbouali University of Chlef, Ouled Fares P.O. Box 78, 02180, Chlef, Algeria \\ ${ }^{2}$ Laboratory of Mathematics and its Applications (LAMAP), University of Oran1, Oran, Algeria
}

*Corresponding author: m.zellal@yahoo.fr

\begin{abstract}
In this paper, He's variational iteration method (VIM), established by He in (1999), is adopted to solve two and three dimensional of Navier-Stokes equation in cartesian coordinates. This method is a powerful tool to handle linear and nonlinear models. The main property of the method is its softness and ability to solve nonlinear equations, accurately and easily. Using variational iteration method, it is possible to find the exact solution or a closed approximate solution of a problem. To illustrate the capacity and reliability of this method, some examples and numerical results are provided.
\end{abstract}

\section{INTRODUCTION}

The main aim of this work, is to solve the model of the Navier Stokes equation for an incompressible fluid flow is given as follows $[5,8,17]$.

$$
\begin{cases}U_{t}+(U . \nabla) U=\rho_{0} \nabla^{2} U-\frac{1}{\rho} \nabla p, & \text { on } \quad \Omega \times(0, T), \\ \nabla . U=0, & \text { on } \quad \Omega \times(0, T), \\ U=0, & \text { on } \quad \partial \Omega \times(0, T),\end{cases}
$$

Received May $18^{\text {th }}, 2020$; accepted June $8^{\text {th }}$, 2020; published June 24 ${ }^{\text {th }}, 2020$.

2010 Mathematics Subject Classification. 35N05, 35Q30, 65A05, 65N25, 65Q20.

Key words and phrases. Navier-Stokes equation; He's variational iteration method; Lagrange multiplier; correction functional; exact solution; approximate solution.

(C)2020 Authors retain the copyrights of their papers, and all open access articles are distributed under the terms of the Creative Commons Attribution License. 
where $U=(u, v, w), t, p$, denote the fluid vector at the point $(x ; y ; z)$, time and the pressure, respectively, $\Omega \subset \mathbb{R}^{3}$ and $\partial \Omega$ its boundary, $\rho$ is the density, $\rho_{0}$ denotes the kinematic viscosity of the flow.

In Cartesian coordinates, the (3D) Navier-Stokes equation becomes:

$$
\left\{\begin{aligned}
u_{t}+u u_{x}+v u_{y}+w u_{z} & =\rho_{0}\left(u_{x x}+u_{y y}+u_{z z}\right)-\frac{1}{\rho} p_{x}, \\
v_{t}+u v_{x}+v v_{y}+w v_{z} & =\rho_{0}\left(v_{x x}+v_{y y}+v_{z z}\right)-\frac{1}{\rho} p_{y} \\
w_{t}+u w_{x}+v w_{y}+w w_{z} & =\rho_{0}\left(w_{x x}+w_{y y}+w_{z z}\right)-\frac{1}{\rho} p_{z} .
\end{aligned}\right.
$$

Various kinds of analytical methods and numerical methods were used to solve Navier-Stokes equation, as Adomian decomposition method [2,17], fractional reduced differential transformation method [5], modified Laplace decomposition method [15], the meshless local Petrov-Galerkin method [22], homotopy perturbation method [23]. The organization of this paper is as follows:

In Section 2, we review the procedure of He's variational iteration method. In Section 3, we show in some examples and numerical results that the present method gives the exact solution or a very good approximation of the exact solution, even for a small $n$, to illustrate the method, its accuracy, effectiveness and simplicity. A conclusion is given in Section 4.

\section{HE'S VARIATIONAL ITERATION METHOD}

Variational iteration method was first proposed by the Chinese mathematician He ( [9]- [14]). This method has been employed to solve a large variety of linear and nonlinear problems which has various applications in science and engineering, with approximations converging rapidly to accurate solutions.

This approach is successfully and effectively applied to various equations such as Burger's and coupled Burger's equations $[1,4]$. This technique is also employed to solve multispecies Lotka-Volterra equations in [3], the Cauchy reaction-diffusion problem and several test examples are given in [6], in [7] the applications of the present method to solve the Fokker-Planck equation is provided. Author of [9] investigated (VIM) for solving delay differential equations. He's variational iteration method is used in [10] to give the solution of blasius equation, autonomous ordinary differential equations [11], in [12] the variational iteration method is used to construct solitary solutions and compacton-like solutions for nonlinear dispersive equations. Also this procedure is investigated in [16] for solving Helmholtz equation, for solving integro-differential equations [19]. Authors of [20] employed the variational iteration method for solving a parabolic inverse problem. Wazwaz has used this method for handling linear and nonlinear diffusion equations [24], for solving linear and nonlinear systems of PDEs [25], for rational solutions for $\mathrm{KdV}, \mathrm{K}(2,2)$, Burger, and cubic Boussinesq equations which has various applications in science and engineering [26]. In [27], for analytic treatment for linear and nonlinear ODEs, the (VIM) modified using He's polynomials to solve biological population model [28]. The convergence of (VIM) is studied in [18,21]. The (VIM) gives rapidly convergent successive approximations of the exact solution if such a solution exists. 
To illustrate the procedure of this approach, we write a system as shown below $[3,25]$

$$
\left\{\begin{array}{l}
L_{t} u+R_{1}(u, v, w)+N_{1}(u, v, w)=f_{1}, \\
L_{t} v+R_{2}(u, v, w)+N_{2}(u, v, w)=f_{2}, \\
L_{t} w+R_{3}(u, v, w)+N_{3}(u, v, w)=f_{3},
\end{array}\right.
$$

subject to initial conditions,

$$
\left\{\begin{array}{l}
u(x, 0)=g_{1}(x) \\
v(x, 0)=g_{2}(x) \\
w(x, 0)=g_{3}(x)
\end{array}\right.
$$

where $L_{t}$ is considered a first-order partial differential operator, $R_{k}$ and $N_{k}, 1 \leq k \leq 3$ are linear and nonlinear operators respectively, and $f_{1}, f_{2}, f_{3}$ source terms. The correction functionals for equations of (2.1) can be written as

$$
\left\{\begin{array}{c}
u_{n+1}(x, t)=u_{n}(x, t)+\int_{0}^{t} \lambda_{1}(\tau)\left[L u_{n}(\tau)+R_{1}\left(\tilde{u}_{n}, \tilde{v}_{n}, \tilde{w}_{n}\right)+N_{1}\left(\tilde{u}_{n}, \tilde{v}_{n}, \tilde{w}_{n}\right)-f_{1}(\tau)\right] d \tau \\
v_{n+1}(x, t)=v_{n}(x, t)+\int_{0}^{t} \lambda_{2}(\tau)\left[L v_{n}(\tau)+R_{2}\left(\tilde{u}_{n}, \tilde{v}_{n}, \tilde{w}_{n}\right)+N_{2}\left(\tilde{u}_{n}, \tilde{v}_{n}, \tilde{w}_{n}\right)-f_{2}(\tau)\right] d \tau \\
w_{n+1}(x, t)=w_{n}(x, t)+\int_{0}^{t} \lambda_{3}(\tau)\left[L w_{n}(\tau)+R_{3}\left(\tilde{u}_{n}, \tilde{v}_{n}, \tilde{w}_{n}\right)+N_{3}\left(\tilde{u}_{n}, \tilde{v}_{n}, \tilde{w}_{n}\right)-f_{3}(\tau)\right] d \tau
\end{array}\right.
$$

where $\lambda_{k}, 1 \leq k \leq 3$, are general Lagrange multipliers, which can be identified optimally via the variational theory, and $\tilde{u}_{n}, \tilde{v}_{n}$, and $\tilde{w}_{n}$ are restricted variations which means $\delta \tilde{u}_{n}=0, \delta \tilde{v}_{n}=0$ and $\delta \tilde{w}_{n}=0$. It is required first to determine the Lagrange multipliers $\lambda_{k}$ that will be identified optimally via integration by parts. The successive approximations $u_{n+1}(x, t), v_{n+1}(x, t), w_{n+1}(x, t), n \geq 0$, of the solutions $u(x, t)$, $v(x, t)$, and $w(x, t)$ will follow immediately upon using the Lagrange multipliers obtained and by using selected functions $u_{0}, v_{0}$ and $w_{0}$. The initial values are usually used for the selected zeroth approximations. With the Lagrange multipliers $\lambda_{k}$ determined, then several approximations $u_{n}(x, t), v_{n}(x, t), w_{n}(x, t), n \geq 1$, can be determined.

Finally, the solutions are given

$$
\left\{\begin{array}{l}
u(x, t)=\lim _{n \rightarrow \infty} u_{n}(x, t), \\
v(x, t)=\lim _{n \rightarrow \infty} v_{n}(x, t), \\
w(x, t)=\lim _{n \rightarrow \infty} w_{n}(x, t) .
\end{array}\right.
$$




\section{Test eXAmples}

Example 3.1. Consider two-dimensional (2D) Navier-Stokes equations: [2, 5].

$$
\left\{\begin{array}{c}
u_{t}+u u_{x}+v u_{y}=\rho_{0}\left(u_{x x}+u_{y y}\right), \\
v_{t}+u v_{x}+v v_{y}=\rho_{0}\left(v_{x x}+v_{y y}\right)
\end{array}\right.
$$

with the initial conditions:

$$
\left\{\begin{array}{c}
u(x, y, 0)=-\sin (x+y) \\
v(x, y, 0)=\sin (x+y)
\end{array}\right.
$$

The correction functional for (3.1) reads

$$
\left\{\begin{array}{l}
u_{n+1}(x, y, t)=u_{n}(x, y, t)+\int_{0}^{t} \lambda_{1}(\tau)\left[\frac{\partial u_{n}(x, y, \tau)}{\partial \tau}+\tilde{u}_{n} \frac{\partial \tilde{u}_{n}}{\partial x}+\tilde{v}_{n} \frac{\partial \tilde{u}_{n}}{\partial y}-\rho_{0}\left(\frac{\partial^{2} \tilde{u}_{n}}{\partial x^{2}}+\frac{\partial^{2} \tilde{u}_{n}}{\partial y^{2}}\right)\right] d \tau \\
v_{n+1}(x, y, t)=v_{n}(x, y, t)+\int_{0}^{t} \lambda_{2}(\tau)\left[\frac{\partial v_{n}(x, y, \tau)}{\partial \tau}+\tilde{u}_{n} \frac{\partial \tilde{v}_{n}}{\partial x}+\tilde{v}_{n} \frac{\partial \tilde{v}_{n}}{\partial y}-\rho_{0}\left(\frac{\partial^{2} \tilde{v}_{n}}{\partial x^{2}}+\frac{\partial^{2} \tilde{v}_{n}}{\partial y^{2}}\right)\right] d \tau
\end{array}\right.
$$

This yields the stationary conditions

$$
\left\{\begin{array}{l}
1+\lambda_{1}=0, \quad \lambda_{1}^{\prime}(\tau=t)=0 \\
1+\lambda_{2}=0, \quad \lambda_{2}^{\prime}(\tau=t)=0 .
\end{array}\right.
$$

As a result we find

$$
\lambda_{1}=\lambda_{2}=-1
$$

Substituting these values of the Lagrange multipliers into the functionals (3.3) gives the iteration formulas

$$
\left\{\begin{array}{l}
u_{n+1}(x, y, t)=u_{n}(x, y, t)-\int_{0}^{t}\left[\frac{\partial u_{n}(x, y, \tau)}{\partial \tau}+u_{n} \frac{\partial u_{n}}{\partial x}+v_{n} \frac{\partial u_{n}}{\partial y}-\rho_{0}\left(\frac{\partial^{2} u_{n}}{\partial x^{2}}+\frac{\partial^{2} u_{n}}{\partial y^{2}}\right)\right] d \tau \\
v_{n+1}(x, y, t)=v_{n}(x, y, t)-\int_{0}^{t}\left[\frac{\partial v_{n}(x, y, \tau)}{\partial \tau}+u_{n} \frac{\partial v_{n}}{\partial x}+v_{n} \frac{\partial v_{n}}{\partial y}-\rho_{0}\left(\frac{\partial^{2} v_{n}}{\partial x^{2}}+\frac{\partial^{2} v_{n}}{\partial y^{2}}\right)\right] d \tau
\end{array}\right.
$$

We can select $u_{0}(x, y, t)=-\sin (x+y), v_{0}(x, y, t)=\sin (x+y)$, by using the given initial values. Accordingly, we obtain the following successive approximations: 


$$
\begin{aligned}
u_{1}(x, y, t) & =-\sin (x+y)\left(1-2 \rho_{0} t\right), \\
v_{1}(x, y, t) & =\sin (x+y)\left(1-2 \rho_{0} t\right), \\
u_{2}(x, y, t) & =-\sin (x+y)\left(1-2 \rho_{0} t+2 \rho_{0}^{2} t^{2}\right), \\
v_{2}(x, y, t) & =\sin (x+y)\left(1-2 \rho_{0} t+2 \rho_{0}^{2} t^{2}\right), \\
u_{3}(x, y, t) & =-\sin (x+y)\left(1-2 \rho_{0} t+2 \rho_{0}^{2} t^{2}-\frac{4}{3} \rho_{0}^{3} t^{3}\right), \\
v_{3}(x, y, t) & =\sin (x+y)\left(1-2 \rho_{0} t+2 \rho_{0}^{2} t^{2}-\frac{4}{3} \rho_{0}^{3} t^{3}\right), \\
\vdots & \\
u_{n}(x, y, t) & =-\sin (x+y)\left(1-2 \rho_{0} t+2 \rho_{0}^{2} t^{2}+\cdots\right), \\
v_{n}(x, y, t) & =\sin (x+y)\left(1-2 \rho_{0} t+2 \rho_{0}^{2} t^{2}+\cdots\right) .
\end{aligned}
$$

The final form of the solution will be as follows

$$
\left\{\begin{aligned}
u(x, y, t) & =-\sin (x+y)\left(1+\left(-2 \rho_{0} t\right)+\frac{\left(-2 \rho_{0} t\right)^{2}}{2 !}+\frac{\left(-2 \rho_{0} t\right)^{3}}{3 !}+\cdots\right) \\
& =-\sin (x+y) e^{-2 \rho_{0} t} \\
v(x, y, t) & =\sin (x+y)\left(1+\left(-2 \rho_{0} t\right)+\frac{\left(-2 \rho_{0} t\right)^{2}}{2 !}+\frac{\left(-2 \rho_{0} t\right)^{3}}{3 !}+\cdots\right) \\
& =\sin (x+y) e^{-2 \rho_{0} t} .
\end{aligned}\right.
$$

Which is an exact solution and is same as obtained by [5].

Table 1: Numerical results when $\rho_{0}=0.5, x=0.1$ and $y=0.5$ in Example 3.1.

\begin{tabular}{ccccccc}
\hline$t$ & $u$ & $u_{3}$ & $\left|u-u_{3}\right|$ & $v$ & $v_{3}$ & $\left|v-v_{3}\right|$ \\
\hline 0 & -0.56464247 & -0.56464247 & 0 & 0.56464247 & 0.56464247 & 0 \\
0.05 & -0.53710453 & -0.53710453 & 0 & 0.53710453 & 0.53710453 & 0 \\
0.1 & -0.51090964 & -0.51090968 & $0.4628 \mathrm{E}-7$ & 0.51090964 & 0.51090968 & $0.4628 \mathrm{E}-7$ \\
0.15 & -0.48599228 & -0.48599262 & 0.9719 & 0.48599228 & 0.48599262 & 0.9719 \\
0.2 & -0.46229015 & -0.46229162 & 0.9246 & 0.46229015 & 0.46229162 & 0.9246 \\
0.25 & -0.43974400 & -0.43974841 & 0.8795 & 0.43974400 & 0.43974841 & 0.8795 \\
0.30 & -0.41829743 & -0.41830832 & $0.1089 \mathrm{E}-4$ & 0.41829743 & 0.41830832 & $0.1089 \mathrm{E}-4$ \\
0.35 & -0.39789682 & -0.39792017 & $0.2335 \mathrm{E}-4$ & 0.39789682 & 0.39792017 & $0.2335 \mathrm{E}-4$ \\
0.40 & -0.37849117 & -0.37853631 & $0.4514 \mathrm{E}-4$ & 0.37849117 & 0.37853631 & $0.4514 \mathrm{E}-4$ \\
\hline
\end{tabular}



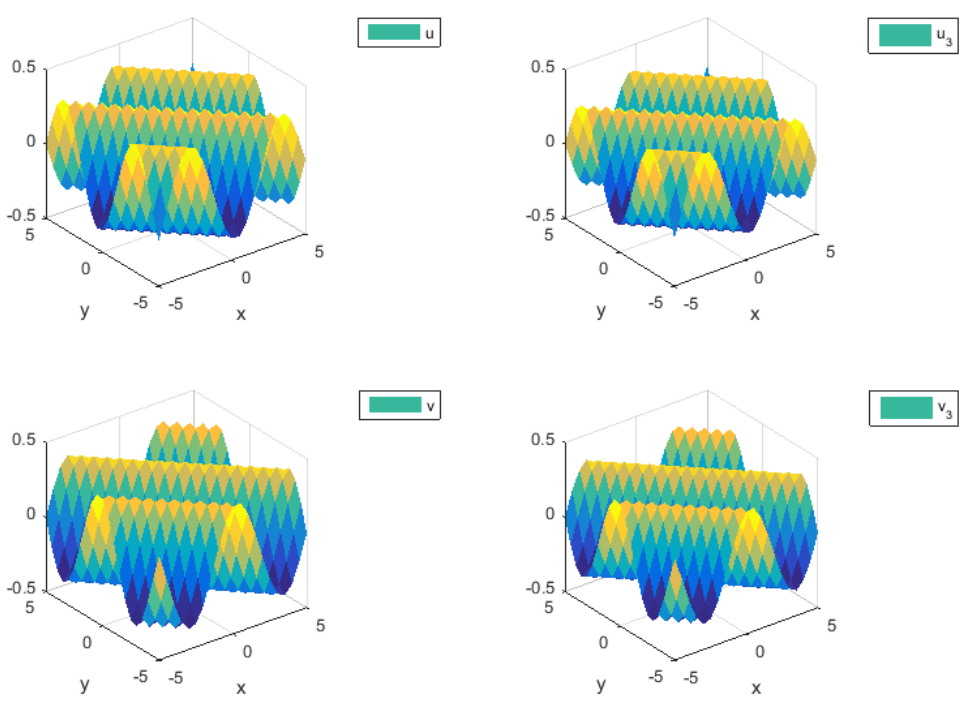

Figure 1. The behavior of $u, u_{3}$ and $v, v_{3}$ in Example 3.1 at $t=1$ with the parameters $\rho_{0}=0,5$.

Example 3.2. Consider two-dimensional (2D) Navier-Stokes equations:

$$
\left\{\begin{array}{l}
u_{t}+u u_{x}+v u_{y}=\rho_{0}\left(u_{x x}+u_{y y}\right), \\
v_{t}+u v_{x}+v v_{y}=\rho_{0}\left(v_{x x}+v_{y y}\right),
\end{array}\right.
$$

with the initial conditions:

$$
\left\{\begin{array}{c}
u(x, y, 0)=-e^{x+y} \\
v(x, y, 0)=e^{x+y} .
\end{array}\right.
$$

We follow the same procedure discussed in Example (3.1), we can select $u_{0}(x, y, t)=-e^{x+y}, v_{0}(x, y, t)=$ $e^{x+y}$ in the iteration formulas(3.6), by using the given initial values. Accordingly, we obtain the following successive approximations: 


$$
\begin{aligned}
u_{1}(x, y, t) & =-e^{x+y}\left(1+2 \rho_{0} t\right) \\
v_{1}(x, y, t) & =e^{x+y}\left(1+2 \rho_{0} t\right) \\
u_{2}(x, y, t) & =-e^{x+y}\left(1+2 \rho_{0} t+2 \rho_{0}^{2} t^{2}\right) \\
v_{2}(x, y, t) & =e^{x+y}\left(1+2 \rho_{0} t+2 \rho_{0}^{2} t^{2}\right) \\
u_{3}(x, y, t) & =-e^{x+y}\left(1+2 \rho_{0} t+2 \rho_{0}^{2} t^{2}+\frac{4}{3} \rho_{0}^{3} t^{3}\right) \\
v_{3}(x, y, t) & =e^{x+y}\left(1+2 \rho_{0} t+2 \rho_{0}^{2} t^{2}+\frac{4}{3} \rho_{0}^{3} t^{3}\right), \\
u_{4}(x, y, t) & =-e^{x+y}\left(1+2 \rho_{0} t+2 \rho_{0}^{2} t^{2}+\frac{4}{3} \rho_{0}^{3} t^{3}+\frac{2}{3} \rho_{0}^{4} t^{4}\right), \\
v_{4}(x, y, t) & =e^{x+y}\left(1+2 \rho_{0} t+2 \rho_{0}^{2} t^{2}+\frac{4}{3} \rho_{0}^{3} t^{3}+\frac{2}{3} \rho_{0}^{4} t^{4}\right), \\
\vdots & \\
v_{n}(x, y, t) & =e^{x+y}\left(1+\left(2 \rho_{0} t\right)+\frac{\left(2 \rho_{0} t\right)^{2}}{2 !}+\cdots\right) . \\
u_{n}(x, y, t) & =-e^{x+y}\left(1+\left(2 \rho_{0} t\right)+\frac{\left(2 \rho_{0} t\right)^{2}}{2 !}+\cdots\right),
\end{aligned}
$$

Finally, the exact solution may be obtained as follows

which are exact solutions [5].

$$
\left\{\begin{array}{c}
u(x, y, t)=-e^{x+y} e^{2 \rho_{0} t}=-e^{x+y+2 \rho_{0} t}, \\
v(x, y, t)=e^{x+y} e^{2 \rho_{0} t}=e^{x+y+2 \rho_{0} t}
\end{array}\right.
$$

Consider the following tables 2 and 3 with the observation that $v=-u$.

Table 2: Numerical results when $\rho_{0}=1, x=0.1$ and $y=0.5$ in Example 3.2.

\begin{tabular}{cccc}
\hline$t$ & $u$ & $u_{4}$ & $\left|u-u_{4}\right|=\left|v-v_{4}\right|$ \\
\hline 0 & -1.8221188 & -1.8221188 & 0 \\
0.05 & -2.0137527 & -2.0137526 & $1.5441055 \mathrm{E}-7$ \\
0.1 & -2.2255409 & -2.2255359 & $5.0256955 \mathrm{E}-6$ \\
0.15 & -2.4596031 & -2.4595643 & $3.8824935 \mathrm{E}-5$ \\
0.2 & -2.7182818 & -2.7181155 & $1.6647662 \mathrm{E}-4$ \\
0.25 & -3.0041660 & -3.0036490 & $5.1706393 \mathrm{E}-4$ \\
0.30 & -3.3201169 & -3.3188072 & $1.3097397 \mathrm{E}-3$ \\
0.35 & -3.6692967 & -3.6664144 & $8.8236261 \mathrm{E}-3$ \\
0.40 & -4.0552000 & -4.0494768 & $5.7231449 \mathrm{E}-3$ \\
\hline
\end{tabular}


Table 3: Absolute error $=\left|u-u_{4}\right|$ when $\rho_{0}=1$ in Example 3.2.

\begin{tabular}{|c|c|c|c|}
\hline$t$ & $t=0.1$ & $t=0.3$ & $t=0.5$ \\
\hline$(x, y)$ & $\left|u-u_{4}\right|$ & $\left|u-u_{4}\right|$ & $\left|u-u_{4}\right|$ \\
\hline$(0.1,0.1)$ & 3.36882444 E-6 & 8.7794477954 E-4 & $1.2151119386 \mathrm{E}-2$ \\
\hline $0.3,0.1)$ & 4.11469146 E-6 & 1.0723241752 E-3 & 1.4841410733 E-2 \\
\hline$(0.5,0.2)$ & 5.55425251 E-6 & $1.4474862325 \mathrm{E}-3$ & 2.0033808995 E-2 \\
\hline$(0.7,0.2)$ & 6.78397933 E-6 & 1.7679636768 E-3 & $2.4469349563 \mathrm{E}-2$ \\
\hline$(0.3,0.3)$ & 5.02569550 E-6 & 1.3097397053 E-3 & $1.8127340004 \mathrm{E}-2$ \\
\hline$(0.5,0.3)$ & 6.13839835 E-6 & 1.5997196885 E-3 & $2.2140783079 \mathrm{E}-2$ \\
\hline$(0.7,0.5)$ & 9.15741425 E-6 & 2.3865013406 E-3 & 3.3030167023 E-2 \\
\hline$(0.9,0.5)$ & 1.11848910 E-5 & 2.9148793198 E-3 & 4.0343137104 E-2 \\
\hline
\end{tabular}

Example 3.3. Consider three-dimensional (3D) Navier-Stokes equations:

$$
\left\{\begin{aligned}
u_{t}+u u_{x}+v u_{y}+w u_{z} & =\rho_{0}\left(u_{x x}+u_{y y}+u_{z z}\right), \\
v_{t}+u v_{x}+v v_{y}+w v_{z} & =\rho_{0}\left(v_{x x}+v_{y y}+v_{z z}\right), \\
w_{t}+u w_{x}+v w_{y}+w w_{z} & =\rho_{0}\left(w_{x x}+w_{y y}+w_{z z}\right),
\end{aligned}\right.
$$

with the initial condition:

$$
\left\{\begin{array}{c}
u(x, y, z, 0)=-\frac{1}{2} x+y+z \\
v(x, y, z, 0)=x-\frac{1}{2} y+z \\
w(x, y, z, 0)=x+y-\frac{1}{2} z
\end{array}\right.
$$

The correction functionals for (3.9) read

$$
\left\{\begin{array}{l}
u_{n+1}(x, y, z, t)=u_{n}+\int_{0}^{t} \lambda_{1}(\tau)\left[\frac{\partial u_{n}}{\partial \tau}+\tilde{u}_{n} \frac{\partial \tilde{u}_{n}}{\partial x}+\tilde{v}_{n} \frac{\partial \tilde{u}_{n}}{\partial y}+\tilde{w}_{n} \frac{\partial \tilde{u}_{n}}{\partial z}-\rho_{0}\left(\frac{\partial^{2} \tilde{u}_{n}}{\partial x^{2}}+\frac{\partial^{2} \tilde{u}_{n}}{\partial y^{2}}+\frac{\partial^{2} \tilde{u}_{n}}{\partial z^{2}}\right)\right] d \tau \\
v_{n+1}(x, y, z, t)=v_{n}+\int_{0}^{t} \lambda_{2}(\tau)\left[\frac{\partial v_{n}}{\partial \tau}+\tilde{u}_{n} \frac{\partial \tilde{v}_{n}}{\partial x}+\tilde{v}_{n} \frac{\partial \tilde{v}_{n}}{\partial y}+\tilde{w}_{n} \frac{\partial \tilde{v}_{n}}{\partial z}-\rho_{0}\left(\frac{\partial^{2} \tilde{v}_{n}}{\partial x^{2}}+\frac{\partial^{2} \tilde{v}_{n}}{\partial y^{2}}+\frac{\partial^{2} \tilde{v}_{n}}{\partial z^{2}}\right)\right] d \tau \\
w_{n+1}(x, y, z, t)=w_{n}+\int_{0}^{t} \lambda_{3}(\tau)\left[\frac{\partial w_{n}}{\partial \tau}+\tilde{u}_{n} \frac{\partial \tilde{w}_{n}}{\partial x}+\tilde{v}_{n} \frac{\partial \tilde{w}_{n}}{\partial y}+\tilde{w}_{n} \frac{\partial \tilde{w}_{n}}{\partial z}-\rho_{0}\left(\frac{\partial^{2} \tilde{w}_{n}}{\partial x^{2}}+\frac{\partial^{2} \tilde{w}_{n}}{\partial y^{2}}+\frac{\partial^{2} \tilde{w}_{n}}{\partial z^{2}}\right)\right] d \tau
\end{array}\right.
$$

The stationary conditions are thus given by

$$
\left\{\begin{array}{l}
1+\lambda_{1}=0, \quad \lambda^{\prime}{ }_{1}(\tau=t)=0 \\
1+\lambda_{2}=0, \quad \lambda^{\prime}{ }_{2}(\tau=t)=0 \\
1+\lambda_{3}=0, \quad \lambda^{\prime}{ }_{3}(\tau=t)=0
\end{array}\right.
$$

so that, the Lagrange multipliers can be identified as follows:

$$
\lambda_{1}=\lambda_{2}=\lambda_{3}=-1 \text {. }
$$


He's variational iteration method consists of the following scheme:

$$
\left\{\begin{array}{l}
u_{n+1}(x, y, z, t)=u_{n}-\int_{0}^{t}\left[\frac{\partial u_{n}}{\partial \tau}+u_{n} \frac{\partial u_{n}}{\partial x}+v_{n} \frac{\partial u_{n}}{\partial y}+w_{n} \frac{\partial u_{n}}{\partial z}-\rho_{0}\left(\frac{\partial^{2} u_{n}}{\partial x^{2}}+\frac{\partial^{2} u_{n}}{\partial y^{2}}+\frac{\partial^{2} u_{n}}{\partial z^{2}}\right)\right] d \tau \\
v_{n+1}(x, y, z, t)=v_{n}-\int_{0}^{t}\left[\frac{\partial v_{n}}{\partial \tau}+u_{n} \frac{\partial v_{n}}{\partial x}+v_{n} \frac{\partial v_{n}}{\partial y}+w_{n} \frac{\partial v_{n}}{\partial z}-\rho_{0}\left(\frac{\partial^{2} v_{n}}{\partial x^{2}}+\frac{\partial^{2} v_{n}}{\partial y^{2}}+\frac{\partial^{2} v_{n}}{\partial z^{2}}\right)\right] d \tau \\
w_{n+1}(x, y, z, t)=w_{n}-\int_{0}^{t}\left[\frac{\partial w_{n}}{\partial \tau}+u_{n} \frac{\partial w_{n}}{\partial x}+v_{n} \frac{\partial w_{n}}{\partial y}+w_{n} \frac{\partial w_{n}}{\partial z}-\rho_{0}\left(\frac{\partial^{2} w_{n}}{\partial x^{2}}+\frac{\partial^{2} w_{n}}{\partial y^{2}}+\frac{\partial^{2} w_{n}}{\partial z^{2}}\right)\right] d \tau
\end{array}\right.
$$

Starting with initial approximations: $u_{0}(x, y, z, t)=-\frac{1}{2} x+y+z, v_{0}(x, y, z, t)=x-\frac{1}{2} y+z$, and $w_{0}(x, y, z, t)=$ $x+y-\frac{1}{2} z$, from (3.14), other terms of the sequence are computed as follows:

$$
\begin{aligned}
& \left\{\begin{array}{l}
u_{1}(x, y, z, t)=-\frac{1}{2} x+y+z-\frac{9}{4} x t \\
v_{1}(x, y, z, t)=x-\frac{1}{2} y+z-\frac{9}{4} y t \\
w_{1}(x, y, z, t)=x+y-\frac{1}{2} z-\frac{9}{4} z t
\end{array}\right. \\
& \left\{\begin{array}{l}
u_{2}(x, y, z, t)=-\frac{1}{2} x+y+z+\left(-\frac{1}{2} x+y+z\right) \frac{9}{4} t^{2}-\frac{9}{4} x t-\frac{27}{16} x t^{3} \\
v_{2}(x, y, z, t)=x-\frac{1}{2} y+z+\left(x-\frac{1}{2} y+z\right) \frac{9}{4} t^{2}-\frac{9}{4} y t-\frac{27}{16} y t^{3} \\
w_{2}(x, y, z, t)=x+y-\frac{1}{2} z+\left(x+y-\frac{1}{2} z\right) \frac{9}{4} t^{2}-\frac{9}{4} z t-\frac{27}{16} z t^{3}
\end{array}\right. \\
& \left\{\begin{aligned}
u_{3}(x, y, z, t) & =\left(-\frac{1}{2} x+y+z\right)+\left(-\frac{1}{2} x+y+z\right) \frac{9}{4} t^{2}+\left(-\frac{1}{2} x+y+z\right) \frac{27}{8} t^{4}+\left(-\frac{1}{2} x+y+z\right) \frac{81}{64} t^{6} \\
& -\frac{9}{4} x t-\frac{81}{16} x t^{3}-\frac{243}{64} x t^{5}-\frac{729}{1792} x t^{7} \\
v_{3}(x, y, z, t) & =\left(x-\frac{1}{2} y+z\right)+\left(x-\frac{1}{2} y+z\right) \frac{9}{4} t^{2}+\left(x-\frac{1}{2} y+z\right) \frac{27}{8} t^{4}+\left(x-\frac{1}{2} y+z\right) \frac{81}{64} t^{6} \\
& -\frac{9}{4} y t-\frac{81}{16} y t^{3}-\frac{243}{64} y t^{5}-\frac{729}{1792} y t^{7} \\
w_{3}(x, y, z, t) & =\left(x+y-\frac{1}{2} z\right)+\left(x+y-\frac{1}{2} z\right) \frac{9}{4} t^{2}+\left(x+y-\frac{1}{2} z\right) \frac{27}{8} t^{4}+\left(x+y-\frac{1}{2} z\right) \frac{81}{64} t^{6} \\
& -\frac{9}{4} z t-\frac{81}{16} z t^{3}-\frac{243}{64} z t^{5}-\frac{729}{1792} z t^{7}
\end{aligned}\right.
\end{aligned}
$$




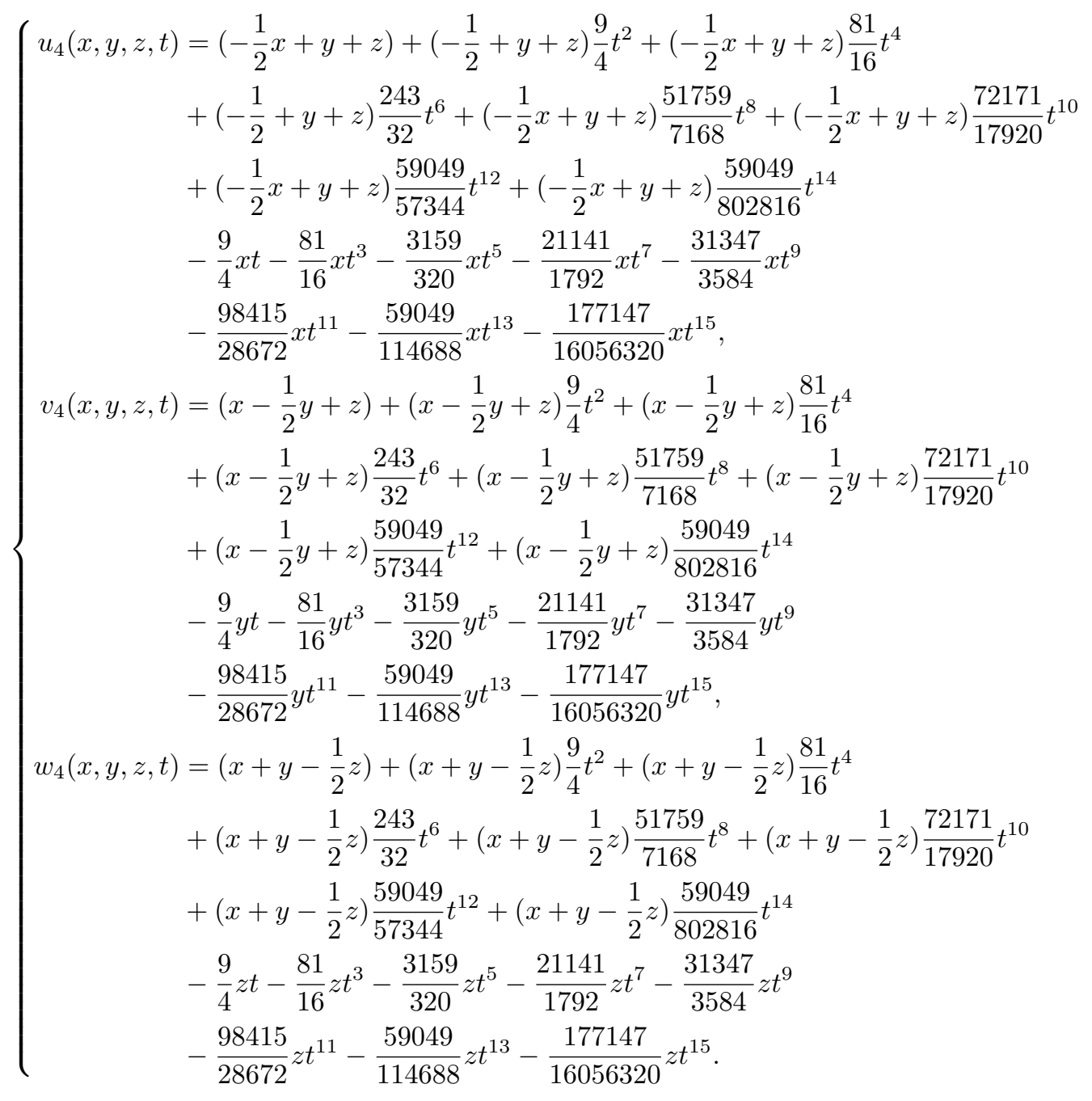

Therefore,

$$
\left\{\begin{aligned}
u(x, y, z, t) & =\left(-\frac{1}{2} x+y+z\right)\left(1+\left(\frac{9}{4}\right) t^{2}+\left(\frac{9}{4}\right)^{2} t^{4}+\cdots\right)-\frac{9}{4} x t\left(1+\left(\frac{9}{4}\right) t^{2}+\left(\frac{9}{4}\right)^{2} t^{4}+\cdots\right) \\
& =\frac{-\frac{1}{2} x+y+z-\frac{9}{4} x t}{1-\frac{9}{4} t^{2}}, \\
v(x, y, z, t) & =\left(x-\frac{1}{2} y+z\right)\left(1+\left(\frac{9}{4}\right) t^{2}+\left(\frac{9}{4}\right)^{2} t^{4}+\cdots\right)-\frac{9}{4} y t\left(1+\left(\frac{9}{4}\right) t^{2}+\left(\frac{9}{4}\right)^{2} t^{4}+\cdots\right) \\
& =\frac{x-\frac{1}{2} y+z-\frac{9}{4} y t}{1-\frac{9}{4} t^{2}} \\
w(x, y, z, t) & =\left(x+y-\frac{1}{2} z\right)\left(1+\left(\frac{9}{4}\right) t^{2}+\left(\frac{9}{4}\right)^{2} t^{4}+\cdots\right)-\frac{9}{4} z t\left(1+\left(\frac{9}{4}\right) t^{2}+\left(\frac{9}{4}\right)^{2} t^{4}+\cdots\right) \\
& =\frac{x+y-\frac{1}{2} z-\frac{9}{4} z t}{1-\frac{9}{4} t^{2}}
\end{aligned}\right.
$$

The same result as [5]. 
Table 4: The (VIM) results for $u$ and $u_{4}$ approximations in Example 3.3

\begin{tabular}{ccccccc}
\hline$t$ & $t=0.01$ & \multicolumn{3}{c}{$t=0.3$} \\
\hline$(x, y, z)$ & $u_{4}$ & $\left|u-u_{4}\right|$ & $u_{4}$ & $\left|u-u_{4}\right|$ & $u_{4}$ & $\left|u-u_{4}\right|$ \\
\hline$(0.1,0.1,0.2)$ & 0.2478057563 & $1.44 \mathrm{e}-11$ & 0.2285415892 & $2.99 \mathrm{e}-4$ & 0.2897586783 & $2.45 \mathrm{e}-2$ \\
$(0.3,0.1,0.2)$ & 0.1432822385 & $4.50 \mathrm{e}-11$ & -0.0640993439 & $1.73 \mathrm{e}-3$ & -0.3486963088 & $7.99 \mathrm{e}-2$ \\
$(0.5,0.2,0.3)$ & 0.2388037309 & $7.51 \mathrm{e}-11$ & -0.1068322397 & $2.89 \mathrm{e}-3$ & -0.5811605148 & $1.33 \mathrm{e}-1$ \\
$(0.7,0.2,0.3)$ & 0.1342802131 & $1.06 \mathrm{e}-10$ & -0.3994731729 & $4.92 \mathrm{e}-3$ & -1.2196155019 & $2.36 \mathrm{e}-1$ \\
$(0.9,0.3,0.4)$ & 0.2298017055 & $1.36 \mathrm{e}-10$ & -0.4422060687 & $6.07 \mathrm{e}-3$ & -1.4520797078 & $2.91 \mathrm{e}-1$ \\
$(0.6,0.3,0.4)$ & 0.3865869822 & $8.97 \mathrm{e}-11$ & -0.0032446693 & $3.03 \mathrm{e}-3$ & -0.4943972270 & $1.34 \mathrm{e}-1$ \\
$(0.8,0.4,0.5)$ & 0.4821084745 & $1.20 \mathrm{e}-10$ & -0.0459775653 & $4.18 \mathrm{e}-3$ & -0.7268614329 & $1.87 \mathrm{e}-1$ \\
$(0.9,0.4,0.5)$ & 0.4298467157 & $1.35 \mathrm{e}-10$ & -0.1922980316 & $5.19 \mathrm{e}-3$ & -1.0460889265 & $2.40 \mathrm{e}-1$
\end{tabular}

Table 5: The (VIM) results for $v$ and $v_{4}$ approximations in Example 3.3.

\begin{tabular}{ccccccc}
\hline \multicolumn{1}{c}{$t=0.01$} & \multicolumn{5}{c}{$t=0.3$} \\
\hline$(x, y, z)$ & $v_{4}$ & $\left|v-v_{4}\right|$ & $v_{4}$ & $\left|v-v_{4}\right|$ & $v_{4}$ & $\left|v-v_{4}\right|$ \\
\hline$(0.1,0.1,0.2)$ & 0.2478057563 & $1.43 \mathrm{e}-11$ & 0.2285415892 & $2.99 \mathrm{e}-4$ & 0.2897586783 & $2.45 \mathrm{e}-2$ \\
$(0.3,0.1,0.2)$ & 0.4478507665 & $1.35 \mathrm{e}-11$ & 0.4784496263 & $1.17 \mathrm{e}-3$ & 0.6957494596 & $7.57 \mathrm{e}-2$ \\
$(0.5,0.2,0.3)$ & 0.6956565227 & $2.77 \mathrm{e}-11$ & 0.7069912153 & $1.47 \mathrm{e}-3$ & 0.9855081381 & $1.00 \mathrm{e}-1$ \\
$(0.7,0.2,0.3)$ & 0.8957015329 & $2.70 \mathrm{e}-11$ & 0.9568992524 & $2.35 \mathrm{e}-3$ & 1.3914989194 & $1.51 \mathrm{e}-1$ \\
$(0.9,0.3,0.4)$ & 1.1435072892 & $4.12 \mathrm{e}-11$ & 1.1854408415 & $2.65 \mathrm{e}-3$ & 1.6812575977 & $1.76 \mathrm{e}-1$ \\
$(0.6,0.3,0.4)$ & 0.8434397739 & $4.24 \mathrm{e}-11$ & 0.8105787860 & $1.33 \mathrm{e}-3$ & 1.0722714258 & $9.92 \mathrm{e}-2$ \\
$(0.8,0.4,0.5)$ & 1.0912455303 & $5.66 \mathrm{e}-11$ & 1.0391203750 & $1.63 \mathrm{e}-3$ & 1.3620301041 & $1.24 \mathrm{e}-1$ \\
$(0.9,0.4,0.5)$ & 1.1912680354 & $5.63 \mathrm{e}-11$ & 1.1640743935 & $2.07 \mathrm{e}-3$ & 1.5650254948 & $1.49 \mathrm{e}-1$ \\
\hline
\end{tabular}


Table 6: The (VIM) results for $w$ and $w_{4}$ approximations in Example 3.3.

\begin{tabular}{ccccccc}
\hline$t$ & $t=0.01$ & \multicolumn{5}{c}{$t=0.3$} \\
\hline$(x, y, z)$ & $w_{4}$ & $\left|w-w_{4}\right|$ & $w_{4}$ & $\left|w-w_{4}\right|$ & $w_{4}$ & $\left|w-w_{4}\right|$ \\
\hline$(0.1,0.1,0.2)$ & 0.0955214924 & $3.00 \mathrm{e}-11$ & -0.0427328960 & $1.15 \mathrm{e}-3$ & -0.2324642059 & $5.33 \mathrm{e}-2$ \\
$(0.3,0.1,0.2)$ & 0.2955665025 & $2.93 \mathrm{e}-11$ & 0.2071751411 & $2.79 \mathrm{e}-4$ & 0.1735265755 & $2.09 \mathrm{e}-3$ \\
$(0.5,0.2,0.3)$ & 0.5433722588 & $4.35 \mathrm{e}-11$ & 0.4357167302 & $1.95 \mathrm{e}-5$ & 0.4632852538 & $2.24 \mathrm{e}-2$ \\
$(0.7,0.2,0.3)$ & 0.7434172689 & $4.28 \mathrm{e}-11$ & 0.6856247672 & $8.96 \mathrm{e}-4$ & 0.8692760351 & $7.36 \mathrm{e}-2$ \\
$(0.9,0.3,0.4)$ & 0.9912230253 & $5.70 \mathrm{e}-11$ & 0.9141663564 & $1.19 \mathrm{e}-3$ & 1.1590347135 & $9.81 \mathrm{e}-2$ \\
$(0.6,0.3,0.4)$ & 0.6911555100 & $5.82 \mathrm{e}-11$ & 0.5393043008 & $1.19 \mathrm{e}-4$ & 0.5500485415 & $2.19 \mathrm{e}-2$ \\
$(0.8,0.4,0.5)$ & 0.9389612664 & $7.24 \mathrm{e}-11$ & 0.7678458899 & $1.79 \mathrm{e}-4$ & 0.8398072198 & $4.59 \mathrm{e}-2$ \\
$(0.9,0.4,0.5)$ & 1.0389837714 & $7.20 \mathrm{e}-11$ & 0.8927999085 & $6.17 \mathrm{e}-4$ & 1.0428026105 & $7.15 \mathrm{e}-2$ \\
\hline
\end{tabular}

It can be observed through tables [1-6] that this method is efficient and accurate for different values of time and place.

Conclusion. There are two main objectives for this work. The first presents an alternative approach to variation iteration method to handle non-linear problems. The second is to use this method to solve the twodimensional (2D) and three-dimensional (3D) Navier-Stokes equations in Cartesian coordinates. It is obvious that the method gives several successive approximations through determining the Lagrange multipliers and using the iteration. (VIM) reduces the size of calculations and facilitates the computational work when compared with (ADM) or (HPM) techniques. He's variational iteration method is suitable as an alternative approach to current techniques being employed to a wide variety of problems in physics. The Navier-Stokes equations were examined. The desired solutions were obtained rapidly and in a direct way. The two goals were achieved.

Acknowledgements: The two authors, sincerely thank the editor and the referees for their guidance and suggestions.

Conflicts of Interest: The author(s) declare that there are no conflicts of interest regarding the publication of this paper.

\section{References}

[1] M.A. Abdou, A.A. Soliman, Variational iteration method for solving Burger's and coupled Burger's equations, J. Comput. Appl. Math. 181 (2005), 245-251.

[2] A. AlSaif, Analytical approximate solutions for two-dimensional incompressible Navier-Stokes equations, Adv. Phys. Theor. Appl. 49 (2015), 69-86.

[3] B. Batiha, M.S.M. Noorani, I. Hashim, Variational iteration method for solving multispecies Lotka-Volterra equations, Comput. Math. Appl. 54 (2007), 903-909. 
[4] J. Biazar, H. Aminikhah, Exact and numerical solutions for non-linear Burger's equation by VIM, Math. Computer Model. 49 (2009), 1394-1400.

[5] B.K. Singh, P. Kumar, FRDTM for numerical simulation of multi-dimensional, time-fractional model of Navier-Stokes equation, Ain Shams Eng. J. 9 (2018), 827-834.

[6] M. Dehghan, F. Shakeri, Application of He's variational iteration method for solving the Cauchy reaction-diffusion problem, J. Comput. Appl. Math. 214 (2008), 435-446.

[7] M. Dehghan, M. Tatari, The use of He's variational iteration method for solving the Fokker-Planck equation, Phys. Scr. 74 (2006), 310-316.

[8] M. El-Shahed, A. Salem, On the generalized Navier-Stokes equations, Appl. Math. Comput. 156 (2005), $287-293$.

[9] J.H. He, Variational iteration method for delay differential equations, Commun. Nonlinear Sci. Numer. Simul. 2 (4) (1997), $235-236$.

[10] J.H. He, Variational iteration method-A kind of non-linear analytical technique: Some examples, Internat. J. Non-Linear Mech. 34 (1999), 699-708.

[11] J.H. He, Variational iteration method for autonomous ordinary differential systems, Appl. Math. Comput. 114 (2/3) (2000), $115-123$.

[12] J.H. He, X.H. Wu, Construction of solitary solution and compacton-like solution by variational iteration method, Chaos Solitons Fractals. 29 (2006), 108-113.

[13] J. H. He, Variational iteration method-Some recent results and new interpretations, J. Comput. Appl. Math. 207 (2007), $3-17$.

[14] J. H. He,, X. H. Wu, Variational iteration method: New development and applications, Comput. Math. Appl. 54 (7-8) (2007), 881-894.

[15] S. Kumar, D. Kumar, S. Abbasbandy, and M. M. Rashidi, Analytical Solution of Fractional Navier-Stokes Equation by using Modified Laplace Decomposition Method, Ain Shams Eng. J. 5 (2) (2014), 569-574.

[16] S. Momani, S. Abuasad, Application of He's variational iteration method to Helmholtz equation, Choas Solitons Fractals. 27 (2006), 1119-1123.

[17] S. Momani, Z. Odibat, Analytical solution of a time-fractional Navier-Stokes equation by Adomian decomposition method, Appl. Math. Comput. 177 (2) (2006), 488-494.

[18] Z. M. Odibat, A study on the convergence of variational iteration method, Math. Computer Model. 51 (2010), 1181-1192.

[19] X. Shang, D. Han, Application of the variational iteration method for solving nth-order integro-differential equations, J. Comput. Appl. Math. 234 (2010), 1442-1447.

[20] M. Tatari, M. Dehghan, He's variational iteration method for computing a control parameter in a semi-linear inverse parabolic equation, Chaos Solitons Fractals. 33 (2007), 671-677.

[21] M. Tatari, M. Dehghan, On the convergence of He's variational iteration method, J. Comput. Appl. Math. 207 (2007), $121-128$.

[22] N. Thamareerat, A. Luadsong, N. Aschariyaphotha, The meshless local Petrov-Galerkin method based on moving Kriging interpolation for solving the time fractional Navier-Stokes equations, SpringerPlus. 5 (2016), 417.

[23] H. A. Wahab, J. Anwar, B. Saira, N. Muhammad, S. Muhammad and H. Sajjad, Application of homotopy perturbation method to the Navier-Stokes equations in cylindrical coordinates, Comput. Ecol. Softw. 5 (2) (2015), 139-151.

[24] A. M. Wazwaz, The variational iteration method: A powerful scheme for handling linear and nonlinear diffusion equations, Comput. Math. Appl. 54 (2007), 933-939. 
[25] A. M. Wazwaz, The variational iteration method for solving linear and nonlinear systems of PDEs, Comput. Math. Appl. 54 (2007), 895-902.

[26] A. M. Wazwaz, The variational iteration method for rational solutions for $\mathrm{KdV}, \mathrm{K}(2,2)$, Burgers, and cubic Boussinesq equations, J. Comput. Appl. Math. 207 (2007), 18-23.

[27] A. M. Wazwaz, The variational iteration method for analytic treatment for linear and nonlinear ODEs, Appl. Math. Comput. 212 (2009), 120-134.

[28] M. Zellal, K. Belghaba, An accurate algorithm for solving biological population model by the variational iteration method using He's polynomials, Arab J. Basic Appl. Sci. 25 (3) (2018), 142-149. 